\title{
Stepwise chromosome evolution in Drosophila albomicans
}

\author{
YUH-CHYN YU†, FEI-JANN LIN $\$$ \& HWEI-YU CHANG* $+\$$ \\ $\uparrow$ Department of Entomology, National Taiwan University, Taipei, Taiwan 106, R.O.C. and $\ddagger$ Institute of Zoology, \\ Academia Sinica, Nankang, Taipei, Taiwan 115, R.O.C.
}

\begin{abstract}
A 'stepwise chromosome evolution model' is proposed to elaborate the two stages of the sex chromosome evolution of Drosophila albomicans. To explain the occurrence of both a 3-X and a 3-Y in this species it is proposed that these chromosomes evolved sequentially. The selection forces acting in the two stages are different. The genic composition in the first fused sex chromosome of D. albomicans was supposed to be selectively superior to the primitive unfused chromosomes, thus the fused chromosome could overcome the meiotic structural incompatibility and be maintained in the population during the first stage. When the second fusion occurred, the newly fused sex chromosome was preferentially selected, as it could provide a meiotic pairing partner for the first fusion. The fused sex chromosomes were eventually fixed in the population. Based on this model, we tried to determine which fused chromosome, 3-X or 3-Y, established first. The karyotyping result of four experimental hybrid populations showed that the fused $3-X$, as opposed to $3-Y$, chromosome has higher fitness values. However, without the coexistence of $3-\mathrm{X}, 3-\mathrm{Y}$ is excluded rapidly. Therefore, $3-\mathrm{X}$ is likely to be the first fused chromosome in the stepwise chromosome evolution model. The formation and fixation of the fused 3-Y chromosome occurred subsequently.
\end{abstract}

Keywords: chromosome evolution, Drosophila albomicans, hybridization, karyotype selection.

\section{Introduction}

Drosophila albomicans belongs to the $D$. nasuta subgroup of the D. immigrans species group. Species within this subgroup have similar morphology but with varying degrees of reproductive isolation (Kitagawa et al., 1982). Drosophila albomicans (Duda, 1923) is distributed from south-east Asia to north-eastern India, including Okinawa, Taiwan, India, Malaysia and southern China. It is one of the most common drosophilids in this area. In the last decade, D. albomicans has invaded the Japanese mainland and spread to western Japan (Ohsako et al., 1994). Its sibling species $D$. nasuta (Lamb, 1914) is found in the eastern coast of tropical Africa, Madagascar, the Seychelles, Sri Lanka, and most of the Indian subcontinent. David \& Tsacas (1980) reported that D. nasuta has been passively transported to the West Coast of Africa, including Congo, Cameroon and Dahomey. Although these two species may have a chance of coming into contact with each other in the north-east part of India according to their distribution, they are considered as allopatric species because no direct information of

*Correspondence. Department of Entomology, National Taiwan University, Taipei, Taiwan 106, R.O.C.

E-mail: hwei@ccms.ntu.edu.tw sympatry has ever been reported and no hybrids have ever been found in nature near the border.

Meera \& Ranganath (1991) have extensively reviewed the karyotypic evolution in the immigrans species group of Drosophila. They classified 34 representative karyotypes of the immigrans group (from $2 n=12$ to $2 n=6$ ) into six successive stages. They also suggested that centric fusions played a major role in the evolutionary divergence of the karyotypes of the immigrans species group of Drosophila. Drosophila albomicans and D. nasuta have different karyotypes as shown in our previous report (Yu et al., 1997). The karyotype of a D. nasuta $(2 n=8)$ male consists of an acrocentric $\mathrm{X}$ chromosome, a submetacentric (J-shaped) Y chromosome, a pair of metacentric chromosome 2, a pair of large acrocentric chromosome 3 , and a pair of small dot-like chromosome 4. Male $D$. albomicans $(2 n=6)$ have a pair of metacentric chromosome 2, a pair of small dot-like chromosome 4, and a pair of large metacentric chromosomes, one of which represents a fusion between ancestral acrocentric chromosome 3 and the $\mathrm{X}$ chromosome and the other a fusion between chromosome 3 and the $\mathrm{Y}$ chromosome (i.e. 3-X and 3-Y). The occurrence of two rare events of centric fusion causing $60 \%$ of the $D$. albomicans genome to be sex-linked is indeed a very special case. 
The high chromosomal inversion polymorphism (Lin et al., 1974) and shared inversion types in D. albomicans with D. nasuta (Ranganath \& Krishnamurthy, 1975; Kumar \& Gupta, 1986; Singh \& Kalisch, 1991) suggest that D. albomicans might not have experienced a long bottleneck during its speciation. Because the fixation of a chromosome alteration could not be simply explained by random genetic drift, we therefore propose a stepwise karyotypic selection model to explain the process of chromosome evolution in D.albomicans. The first stage of chromosome evolution started from the successful fusion of a chromosome 3 to one of the two ancestral sex chromosomes. The fused sex chromosome must have a selective advantage to overcome the meiotic structural incompatibility, otherwise it would not have been maintained in the population. When the second fusion occurred it would have been selected, as it could provide a meiotic pairing partner for the first fusion. Therefore, the pair of fused sex chromosomes will be subsequently fixed in the population. To explore the possible sequence of this stepwise chromosome evolution model, we attempted to reconstruct two alternative sequences of chromosomal fusion by hybridization experiments in the laboratory. Four types of experimental hybrid populations were established to understand which fusion, $3-X$ or $3-Y$, may have come first.

\section{Materials and methods}

\section{Drosophila stocks}

Three Drosophila albomicans and three D. nasuta isofemale stocks were kindly provided by Dr O. Kitagawa. Three D. albomicans stocks (no. 163.5, no. 160.3 and no. 161.3) were collected from Okinawa (Japan), Nokhon Nayok and Chiang Mai (Thailand), respectively. One D. nasuta stock (no. 193.7) was obtained from Mysore (India) and two (no. 193.8 and no. 252.21) from Mombasa (Kenya). Each stock was maintained in a group of four culture vials with standard corn meal Drosophila medium at $22^{\circ} \mathrm{C}$. In each generation, the progenies from four vials were intermingled and randomly redistributed into four fresh vials every 20 days.

A hybrid stock (H23), derived from hybridization of Japan D. albomicans (no. 163.5) and India D. nasuta (no. 193.7), was adopted for construction of certain experimental populations. This stock has fixed sex chromosomes, i.e. $2 n=7$ in males (a primitive chromosome 3, a primitive $\mathrm{Y}$ chromosome, and a fused 3-X chromosome) and $2 n=6$ in females (two fused $3-\mathrm{X}$ chromosomes), and apparently no sex ratio distortion (Yu et al., 1997).

\section{Establishment of experimental hybrid populations}

The hybridization was performed as previously described (Yu et al., 1997). In brief, flies were sexed within $8 \mathrm{~h}$ after emergence, and 3-day-old virgins were used for crosses. Our pretest also showed that 3-day-old flies are sexually mature. All of the experimental populations were raised with nonoverlapping generations in a $25^{\circ} \mathrm{C}$ incubator. The developmental time of D. nasuta is shorter than that of $D$. albomicans. This difference can be properly manipulated during the process of establishing hybrid populations.

To analyse which fusion, $3-\mathrm{X}$ or $3-\mathrm{Y}$, might be established first during evolution, we constructed four types of hybrid populations by crossing D. albomicans and D. nasuta. A scheme for the establishment of these four types of hybrid populations is illustrated in Fig. 1. To avoid bias, each type of hybrid population was established three or four times using different stocks of D. nasuta and D. albomicans, such as no. $193.7 \times$ no. 163.5 (set I), no. $193.8 \times$ no. 160.3 (set II), or no. $252.21 \times$ no. 161.3 (set III). Each hybrid line was started from one pair of flies. In the second generation, two pairs of flies were mated. For establishing the neoX types, two ${ }^{*} \mathrm{~F}_{1}$ females were paired with an ${ }^{*} \mathrm{~F}_{1}$ male and a male from the paternal stock, respectively (Fig. 1, neoX-1 and neoX-2). For establishing the neoY types, two females from the maternal stock were paired with an ${ }^{*} \mathrm{~F}_{1}$ male and a male from the maternal stock, respectively (Fig. 1, neoY-1 and neoY-2). Afterwards, 20 virgin offspring (10 males and 10 females) from each mating pair were mixed and raised with nonoverlapping generations. These 40 virgin flies were designated as the ' $\mathrm{P}$ ' generation, a reference generation for the hybrid population. Our pretest showed no mating preferences among the flies at the reference generation in our experimental populations. The karyotype of the $F_{1}$ larvae generated by the reference generation $(\mathrm{P})$ was then analysed. Only those hybrid lines with the competing fused chromosome frequencies close to $50 \%$ were selected for the subsequent experiments. To avoid bias from individual variations, each hybrid population is composed of five or 10 hybrid lines transferred from generation to generation without mixing. The hybrid male from the cross of Okinawa albomicans female and Indian nasuta male was replaced by a male from $\mathrm{H} 23$ during the establishment of neoX-1-I, and neoY-2-I, and the reason will be discussed later.

\section{Analysis of chromosome evolution}

During the further cultivation, five or 10 larvae were sampled from each hybrid line, and for karyotype 


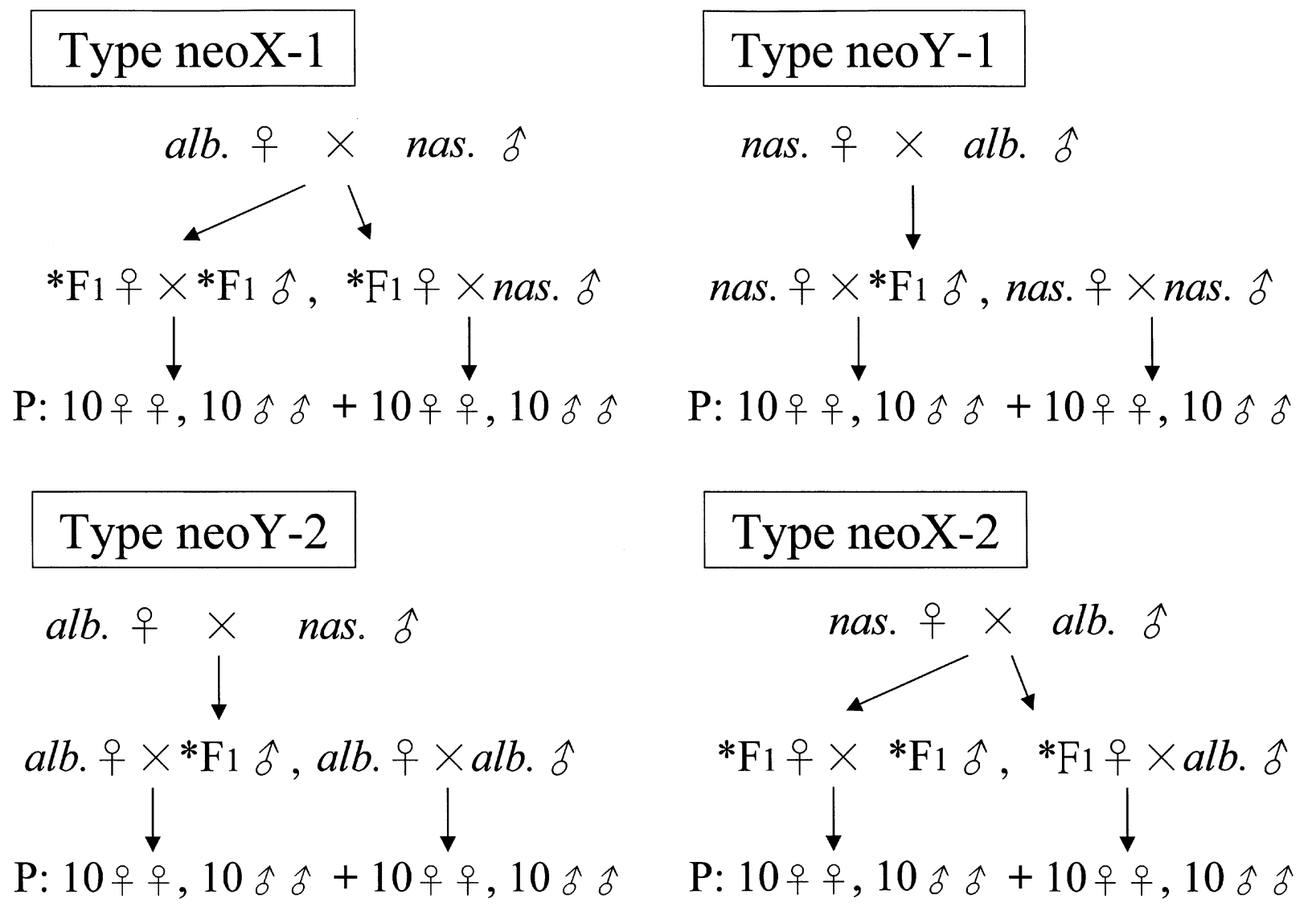

Fig. 1 The protocol for establishing the four experimental hybrid populations. alb., Drosophila albomicans; nas., D. nasuta; ${ }^{*} \mathrm{~F}_{1}$, the offspring of the cross indicated above, not the same as ' $\mathrm{F}_{1}$ ' which are the offspring from the reference generation $(\mathrm{P})$. The bottom line of each population indicates the reference generation. The $* \mathrm{~F}_{1}$ male was replaced by a male from the hybrid stock $\mathrm{H} 23$ while constructing neoX-1-I and neoY-2-I. The reason for this is discussed in the text.

analysis at different generations as indicated in the results. The karyotype of each hybrid population was summarized from all hybrid lines. As described above, each type of hybrid population, except neoY-2, was established three times by using different sets of stocks. There were four neoY-2 populations (designated as neoY-2-I, neoY-2-II, neoY-2-II' and neoY-2-III). The neoY-2-II' population was generated from the same set of stocks as neoY-2-II. At the first stage a population has only one fused chromosome: neoX-1 populations have 3-X and neoY-1 populations have 3-Y; and at the second stage a population (i.e. neoY-2 or neoX-2) has both fused chromosomes $3-X$ and $3-Y$. To find out how the fused sex chromosome behaves against a uniform background of the opposing sex chromosome, the hybrid lines were continuously cultivated for up to 10 generations. From neoX-1 (or neoX-2) populations, we could therefore establish the frequency change of $3-X$ under the fixed 3,Y (or 3-Y) background; and from neoY-1 (or neoY-2) populations, the frequency change of $3-Y$ could be established under fixed $3, \mathrm{X}$ (or $3-\mathrm{X}$ ) background. All possible outcomes for each population are summarized in Table 1.

\section{Karyotype assay}

The karyotypes of the above populations from different generations as indicated in the Results were examined according to a procedure described in $\mathrm{Yu}$ et al. (1997). This method was modified from Guest \& Hsu (1973).

\section{Results}

Four types of hybridization experiments were performed in this report. In each type of hybridization experiment, three sets of populations obtained by crossing different combinations of stocks were used; for instance, type neoX-1 comprises three populations, neoX-1-I, neoX-1II and neoX-1-III. Each population was composed of five or 10 nonmixed lines. The frequencies of sex chromosomes in each population were estimated from all hybrid lines. For investigating the frequency of the 
Table 1 The initial sex chromosome compositions and all possible outcomes of four types of hybrid populations

\begin{tabular}{ccccc}
\hline & Type neoX-1 3,Y & Type neoY-1 3,X & Type neoY-2 3-X & Type neoX-2 3-Y \\
\hline $\mathrm{P}$ & $1 / 2(3, \mathrm{X})+1 / 2(3-\mathrm{X})$ & $1 / 2(3, \mathrm{Y})+1 / 2(3-\mathrm{Y})$ & $1 / 2(3, \mathrm{Y})+1 / 2(3-\mathrm{Y})$ & $1 / 2(3, \mathrm{X})+1 / 2(3-\mathrm{X})$ \\
$\mathrm{F}_{n}$ 3-X fixation or polymorphism & 3-Y fixation or polymorphism & 3-Y fixation & 3-X fixation \\
& 3-X Exclusion & 3-Y Exclusion & 3-Y cannot reach fixation & 3-X cannot reach fixation \\
\hline
\end{tabular}

P: initiation population at the reference generation.

$\mathrm{F}_{n}: n$th generation.

3-X and 3-Y: the fused sex chromosomes.

$3, \mathrm{X}$ and $3, \mathrm{Y}$ : the separate autosome 3 and sex chromosomes.

Table 2 The karyotype assay of type neoX-1 populations of Drosophila albomicans

\begin{tabular}{|c|c|c|c|c|c|}
\hline \multirow[b]{2}{*}{ Set } & \multirow[b]{2}{*}{ Generation } & \multirow[b]{2}{*}{$\mathrm{f}(3-\mathrm{X})( \pm 2 \mathrm{SD})$} & \multicolumn{2}{|c|}{ Sample size } & \multirow[b]{2}{*}{$\mathrm{f}$ (aneuploids) $( \pm 2 \mathrm{SD})$} \\
\hline & & & Male & Female & \\
\hline \multirow{2}{*}{ I } & $\mathrm{F}_{5}$ & $70.0 \%( \pm 5.3 \%)$ & 98 & 102 & $18.5 \%( \pm 5.5 \%)$ \\
\hline & $\mathrm{F}_{10}$ & $84.8 \%( \pm 4.1 \%)$ & 100 & 100 & $14.5 \%( \pm 5.0 \%)$ \\
\hline II & $\mathrm{F}_{10}$ & $47.8 \%( \pm 8.2 \%)$ & 50 & 50 & $9.0 \%( \pm 5.7 \%)$ \\
\hline \multirow[t]{3}{*}{ III } & $\mathrm{F}_{1}$ & $47.3 \%( \pm 11.5 \%)$ & 25 & 25 & $2.0 \%( \pm 4.0 \%)$ \\
\hline & $\mathrm{F}_{5}$ & $50.3 \%( \pm 8.2 \%)$ & 51 & 49 & $3.0 \%( \pm 3.4 \%)$ \\
\hline & $\mathrm{F}_{10}$ & $32.3 \%( \pm 7.7 \%)$ & 53 & 47 & $10.0 \%( \pm 6.0 \%)$ \\
\hline
\end{tabular}

Table 3 The karyotype assay of type neoY-1 populations of Drosophila albomicans

\begin{tabular}{lccrr}
\hline Set & Generation & $\mathrm{f}(3-\mathrm{Y})( \pm 2 \mathrm{SD})$ & Sample size & $\mathrm{f}($ aneuploid $)( \pm 2$ SD) \\
\hline $\mathrm{I}$ & $\mathrm{F}_{1}$ & $50.0 \%(11.3 \%)$ & 79 & $16.5 \%( \pm 8.4 \%)$ \\
& $\mathrm{F}_{5}$ & $12.0 \%( \pm 4.8 \%)$ & 180 & $26.1 \%( \pm 6.5 \%)$ \\
II & $\mathrm{F}_{10}$ & $3.4 \%( \pm 2.7 \%)$ & 50 & $17.8 \%( \pm 5.7 \%)$ \\
& $\mathrm{F}_{1}$ & $33.3 \%( \pm 13.3 \%)$ & 99 & $22.0 \%( \pm 11.7 \%)$ \\
III & $\mathrm{F}_{5}$ & $0 \%( \pm 0 \%)$ & 50 & $14.1 \%( \pm 7.0 \%)$ \\
& $\mathrm{F}_{1}$ & $23.1 \%( \pm 11.9 \%)$ & 100 & $22.0 \%( \pm 11.7 \%)$ \\
\hline
\end{tabular}

3-X chromosome, we sampled five male and five female larvae from each line in the first generation and 10 male and 10 female larvae in the following generations from each of the chosen lines (Tables 2 and 5). For investigating the frequency of the $3-Y$ chromosome, we sampled 10 male larvae from each line in the first generation and 20 male larvae in the following generations (Tables 3 and 4). Because there was no statistically significant discrepancy between different lines within a population, we pooled the data. Confidence intervals of frequencies were provided by \pm 2 standard deviations shown in parentheses (Tables 2-5).

Under the separate $3, \mathrm{Y}$ background, the frequency of the fused 3-X chromosome was maintained after 10 generations (Table 2), i.e. polymorphic at the $F_{10}$ generation. Furthermore, the hybrid populations derived from different geographical origins have distinct trends of frequency changes. The frequency of $3-\mathrm{X}$ rose from $49.6 \%$ in the $F_{1}$ to $84.8 \%$ in the $F_{10}$ in the neoX-1-I population (hybridization from Okinawa D. albomicans and Indian D. nasuta), whereas the frequency dropped from the $\mathrm{F}_{5}(50.3 \%)$ to the $\mathrm{F}_{10}$ $(32.3 \%)$ in the neoX-1-III population (hybridization from Chiang Mai, Thailand D. albomicans and Kenya D. nasuta). In the neoX-1-II population (hybridization from Nakhon Nayok, Thailand D. albomicans and Kenya $D$. nasuta), the frequency of (3-X) remained at a constant level.

(c) The Genetical Society of Great Britain, Heredity, 83, 39-45. 
Table 4 The karyotype assay of type neoY-2 populations of Drosophila albomicans

\begin{tabular}{lcccc}
\hline Set & Generation & $\mathrm{f}(3-\mathrm{Y})( \pm 2 \mathrm{SD})$ & Sample size & $\mathrm{f}$ (aneuploid) $( \pm 2 \mathrm{SD})$ \\
\hline $\mathrm{I}$ & $\mathrm{F}_{1}$ & $56.2 \%( \pm 10.0 \%)$ & 99 & $26.3 \%( \pm 8.8 \%)$ \\
& $\mathrm{F}_{5}$ & $100 \%( \pm 0 \%)$ & 181 & $6.6 \%( \pm 3.7 \%)$ \\
$\mathrm{II}$ & $\mathrm{F}_{1}$ & $49.1 \%( \pm 12.9 \%)$ & 60 & $5.0 \%( \pm 5.6 \%)$ \\
& $\mathrm{F}_{3}$ & $43.9 \%( \pm 9.8 \%)$ & 103 & $4.9 \%( \pm 4.3 \%)$ \\
& $\mathrm{F}_{5}$ & $51.6 \%( \pm 10.0 \%)$ & 100 & $5.0 \%( \pm 4.4 \%)$ \\
& $\mathrm{F}_{10}$ & $52.1 \%( \pm 11.5 \%)$ & 76 & $6.6 \%( \pm 5.7 \%)$ \\
$\mathrm{II}^{\prime}$ & $\mathrm{F}_{1}$ & $48.9 \%( \pm 14.1 \%)$ & 50 & $6.0 \%( \pm 6.7 \%)$ \\
& $\mathrm{F}_{4}$ & $52.8 \%( \pm 10.0 \%)$ & 100 & $1.0 \%( \pm 6.3 \%)$ \\
$\mathrm{III}$ & $\mathrm{F}_{1}$ & $54.5 \%( \pm 14.1 \%)$ & 100 & $1.0 \%( \pm 9.2 \%)$ \\
& $\mathrm{F}_{4}$ & $98.0 \%( \pm 2.8 \%)$ & & $1.0 \%( \pm 2.0 \%)$ \\
\hline
\end{tabular}

Table 5 The karyotype assay of type neoX-2 populations of Drosophila albomicans

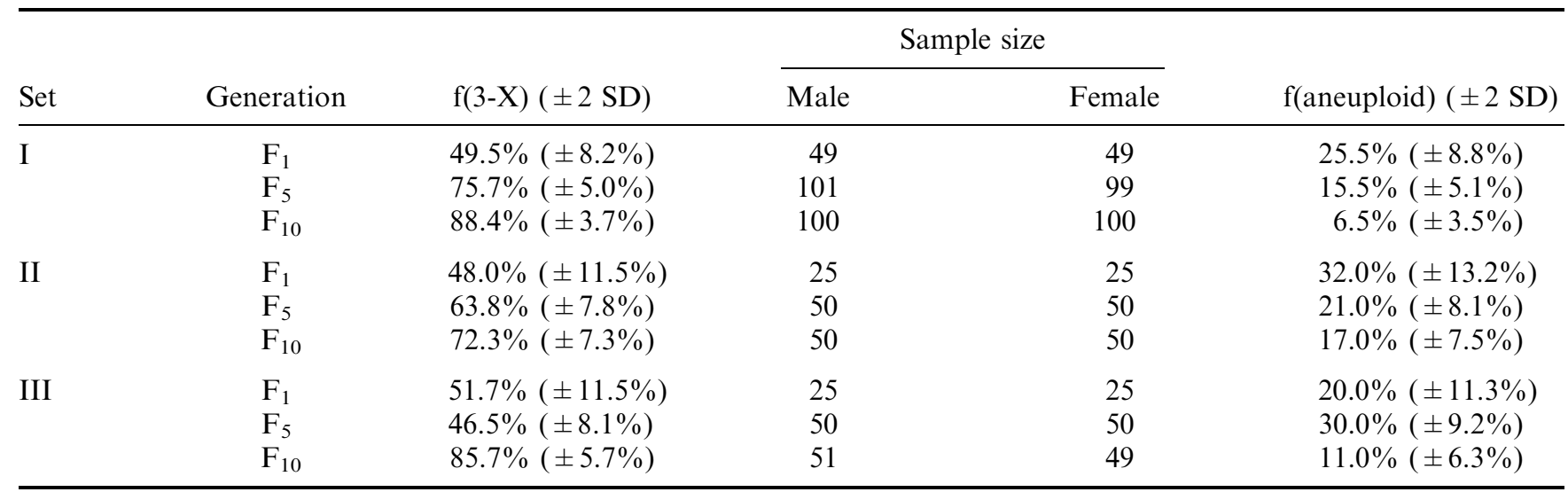

Table 3 shows the changes of karyotype frequency in neoY-1 populations. The frequency of $3-Y$ dropped from $50.0 \%$ in the $F_{1}$ to $3.4 \%$ in the $F_{10}$ in the neoY-1-I population, and in the neoY-1-II and neoY-1-III populations it dropped to 0 within five generations. Under the separate 3,X background, the fused 3-Y chromosome was apparently excluded in all three neoY-1 populations.

Table 4 summarizes the changes of karyotype frequency in neoY-2 populations. Under the fused 3-X background, the fused $3-\mathrm{Y}$ chromosome reached fixation in the neoY-2-I population within five generations, while becoming nearly fixed $(98.0 \%)$ in the neoY-2-III population at the fourth generation. In contrast, polymorphism is still observed among $F_{10}$ progenies in the neoY-2-II population. A repeated experiment (neoY$2-\mathrm{II}^{\prime}$ population) confirmed that the $3-\mathrm{Y}$ chromosome remained polymorphic (at $52.8 \%$ ) in $\mathrm{F}_{4}$ progenies. The rate of chromosome selection in the neoY-2-II population was apparently slower than in both the neoY-2-I and neoY-2-III populations.

The changes of karyotype frequency in the neoX-2 populations are shown in Table 5. In the fused 3-Y background, the frequency of the fused 3-X chromosome increased in all of the three populations (neoX-2-I, neoX-2-II and neoX-2-III).

The results showed that $3-X$ is maintained alone, as are $3-X$ and $3-Y$ together. But when $3-Y$ alone is present, it is not maintained at high frequency. This shows that $3-\mathrm{Y}$ must be associated with $3-\mathrm{X}$ in order to compete effectively.

In addition, aneuploids were frequently observed in the hybrid offspring. The aneuploids include individuals with an extra chromosome 4, males with one or two extra Y chromosomes, or without a Y chromosome, and females with one or two extra $\mathrm{Y}$ chromosomes. The extra $\mathrm{Y}$ chromosome in aneuploids is the one that belongs to D. nasuta. However, the aneuploidy frequencies vary among different populations and in different generations (Tables 2-5).

\section{Discussion}

Apparently the sex chromosome differentiation occurred long before the chromosome fusion in $D$. albomicans. The 
fusion of chromosome 3 to $\mathrm{X}$ and 3 to $\mathrm{Y}$ should be two separate events. Therefore, a two-step fixation of fused chromosomes was required for karyotype evolution of D. albomicans. Although there is a fixed structural difference of chromosomes between $D$. albomicans and $D$. nasuta, their species status is questionable because of the lack of premating isolation (i.e. sexual, mechanical or gametic isolation), and because postmating isolation (i.e. hybrid breakdown) is weak (Chang \& Ayala, 1989). In this report, we focus only on chromosome evolution itself instead of speciation.

Theoretically two kinds of chromosomal selection forces are suggested in our stepwise chromosome evolution model: one is the meiotic structural compatibility of chromosomes, and the other is the selective advantage of a fused chromosome because of its genic composition. However, we can only see the outcome of both forces working together. In stage 1 of chromosome evolution, these two forces are antagonistic to each other. When fusion 1 first occurred, the force of structural incompatibility would have excluded this new chromosomal rearrangement unless it was selectively more advantageous than the unfused state. In stage 2, the second fused chromosome abolished the situation of structural incompatibility in stage 1 . We therefore expect that the first fused chromosome remained polymorphic in stage 1, and that the two fused chromosomes eventually reached fixation in stage 2 .

The production of aneuploids in hybrid populations is evidence for the existence of meiotic structural incompatibility. In stage 1 , aneuploids were maintained (Tables 2 and 3), but the frequency drops significantly, whereas the frequency of the fused chromosome increases in stage 2 (Tables 4 and 5). As in other Drosophila species, aneuploids for larger autosomes (e.g. the second or third chromosomes in this case) were not found and the majority involved the heterochromatic $\mathrm{Y}$ chromosome. All of the $\mathrm{F}_{1}$ hybrids from a cross between $D$. nasuta and D. albomicans are heterokaryotypic but no evidence of reduced fitness was found in this study, nor in our previous study (Chang \& Ayala, 1989). This provides evidence for the existence of a counteracting force to structural incompatibility. These results indicate that the structural heterokaryotype does not necessarily cause problems for its carrier. We suspect that the genic composition of the fused chromosome in stage 1 may play an advantageous role in chromosome segregation or survival.

In our stepwise chromosome evolution model, neoX-1 and neoY-1 populations represented two possibilities at stage 1, whereas neoY-2 and neoX-2 represented populations at stage 2 in the evolution of $D$. albomicans. NeoX1 and neoY-2 populations are two successive stages if 3-X appeared before 3-Y during karyotypic evolution.
NeoY-1 and neoX-2 populations are the alternative. Let us look at the neoY-1 neoX-2 sequence first. The fused 3$\mathrm{Y}$ chromosome was excluded at stage 1 of chromosome evolution in all three neoY-1 populations (Table 3). Although 3-X can be maintained at high frequency in all neoX-2 populations (Table 5), this sequence cannot be correct because of the rapid elimination of 3-Y at the first stage in neoY-1 even if it is given a $50 \%$ frequency to start with. For the neoX-1 neoY-2 sequence, during the first stage, the $3-X$ was maintained (Table 2), and during the second stage, the frequency of 3-Y increased rapidly in neoY-2-I and neoY-2-III populations and was maintained in the neoY-2-II population (Table 4). The results of set I showed that the fused 3-X chromosome had a net selective advantage over the primitive separate $3, \mathrm{X}$ chromosome (Tables 2 and 5), supporting our stepwise chromosome evolution hypothesis. The selective force in set II is not as strong as we expected, but the fused chromosomes were maintained in the population (Table 4). Although the 3-X frequency dropped for the first 10 generations in the neoX-1-III population (Table 2), it is not excluded within five generations, as is 3-Y for the neoY-1-III population (Table 3).

There was a slight difference between set I and the sets II and III during the construction of neoX-1 and neoY-2 populations. The problem is that the sex ratio in the $\mathrm{F}_{2}$ offspring from the cross between an Indian D. nasuta male and an Okinawa $D$. albomicans female is severely distorted (Chang \& Ayala, 1989). This problem was solved by replacing the ${ }^{*} F_{1}$ male with a male from the hybrid stock $\mathrm{H} 23$, which has a fixed karyotype and no sex ratio distortion (Yu et al., 1997). The other two sets of populations do not have the problem of sex ratio distortion.

Whether D. albomicans and D. nasuta are two subspecies or two species is an important issue as a result of the perfectly fertile hybrids produced by them (Nirmala \& Krishnamurthy, 1972; Kitagawa et al., 1982) and the same mate recognition type they share (Lambert, 1982). This systematic problem may be a disadvantage for some investigations, but this could be an advantage for an evolutionary study because it shows that the two taxa are not too far away from the splitting point. They are both widely distributed taxa and the possibility for both of them to survive for a long time is high. It is unlikely that they will produce fertile hybrids at that future time. They will be just like other pairs of species with fixed chromosomal differences, and it will be impossible to determine whether chromosome change is the driving force of their reproductive isolation. The fused $3-\mathrm{X}$ and $3-\mathrm{Y}$ chromosomes are, instead of the cause of reproductive isolation, two correlated mutations fixed in the D. albomicans population. The crosses inevitably involve reconstructed populations that are not identical to the proposed 
original, natural ones because of the time lag. That is why we adopted stocks from different origins to repeat this experiment, and we do find some consistent results. From these results, we can conjecture the track of chromosome evolution in D. albomicans: fusion 1 was the forming of $3-\mathrm{X}$ and fusion 2 was that of 3-Y.

\section{Acknowledgements}

We thank Dr Timothy Prout for valuable comments and for checking the English. This work was supported by the National Science Council of the Republic of China (Grant no. NSC-85-2321-B002-024).

\section{References}

CHANG, H. AND AYALA, F. J. 1989. On the origin of incipient reproductive isolation: the case of Drosophila albomicans and D. nasuta. Evolution, 43, 1614-1624.

DAVID, J. R. AND TSACAS, L. 1980. Cosmopolitan, subcosmopolitan and wide-spread species: Different strategies within the drosophilid family (Diptera). C. r. Soc. Biogeogr., 57, 11-26.

DuDA, O. (1923) Die orientalischen Drosophiliden-Arten des hungarischen National-Museums zu Budapest. Ann. Mus. Nat. Hung., 20, 24-59.

GUEST, w. C. AND HSU, T. C. 1973. A new technique for preparing Drosophila neuroblast chromosomes. Drosoph. Inf. Serv., 50, 193.

KitAGaWA, O., WAKAHAMA, K. I., FUYAMA, Y., SHIMADA, Y., TAKANASHI, E., HATSUMI, M. $E T A L .1982$. Genetic studies of the Drosophila nasuta subgroup, with notes on distribution and morphology. Jap. J. Genet., 57, 113-141.

KUMAR, A. AND GUPTA, J. P. 1986. Inversion polymorphism in Drosophila nasuta. Drosoph. Inf. Serv., 63, 78-80.

LAMB, C. G. 1914. Diptera: Heteroneuridae, Ortalidae, Trypetidae, Sepsidae, Micropezidae, Drosopilidae, Geomyzidae, Milichiidae of the Seychelles. Trans. Linn. Soc. Lond., 16, 307-372.

LAMBERT, D. M. 1982. Mate recognition in members of the Drosophila nasuta complex. Anim. Behav., 30, 438-443.

LIN, F. -J., TSENG, H. C. AND WANG, T. C. 1974. Standard map of the salivary gland chromosomes of Drosophila albomicans Duda. Drosoph. Inf. Serv., 51, 42-43.

MEERA, R. P. AND RANGANATH, H. A. 1991. Karyotype differentiation among members of the immigrans species group of Drosophila. Genetica, 83, 145-152.

NiRmalA, S. S. AND KRISHNAMURThy, N. B. 1972. Drosophila albomicana - a race of Drosophila nasuta. Drosoph. Inf. Serv., 49, 60.

OHSAKo, T., AOTSUKA, T. AND KitAgawa, O. 1994. The origin of the Japanese mainland population of Drosophila albomicans. Jap. J. Genet., 69, 183-194.

RANGANATH, H. A. AND KRISHNAMURTHY, N. B. 1975. Chromosomal polymorphism in Drosophila nasuta. III. Inverted gene arrangements in South Indian populations. J. Hered., 66, 90-96.

SINGH, O. P. AND KALISCH, W. E. 1991. SSP technique applied to EM genome analysis and photo mapping in Drosophila nasuta. Ind. J. Hered., 6, 19-25.

YU, Y. C., LIN, F. -J. AND CHANG, H. 1997. Karyotype polymorphism in hybrid populations of Drosophila nasuta and D. albomicans. Zool. Stud., 35, 251-259. 